
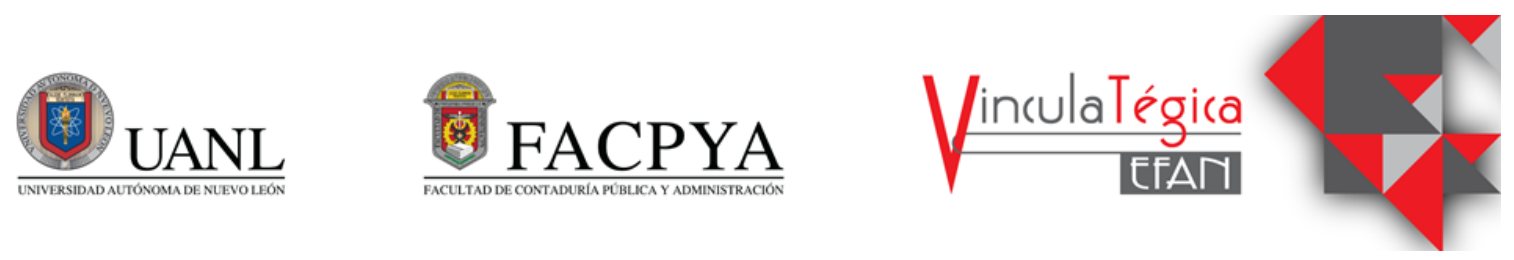

\title{
Estrategias de distribución, sectores minorista y de logística en el ámbito de comercio electrónico
}

\author{
Elda Aurora Aranda Contreras ${ }^{1}$, Sergio Armando Guerra Moya² y Gilberto Rebolledo \\ Iglesias $^{3}$ \\ ${ }^{1}$ Universidad Autónoma de Nuevo León, e_aranda10@yahoo.com, Ave. Universidad s/n Ciudad Universitaria \\ de Nuevo León, 8183294000 \\ ${ }^{21}$ Universidad Autónoma de Nuevo León, sagm52@hotmail.com, Ave. Universidad s/n Ciudad Universitaria \\ de Nuevo León, 8183294000 \\ ${ }^{321}$ Universidad Autónoma de Nuevo León, grebolledoi@yahoo.com.mx, Ave. Universidad s/n Ciudad \\ Universitaria de Nuevo León, 8183294000
}

\author{
Información del artículo revisado por pares \\ Fecha de aceptación: junio-2021 \\ Fecha de publicación en línea: diciembre-2021 \\ DOI: https://doi.org/10.29105/vtga7.2-73
}

\section{Resumen}

El presente estudio es una investigación exploratoria sobre las estrategias que aplican las empresas minoristas que realizan comercio electrónico y de esta forma proveen bienes físicos fundamentalmente y las empresas de logística que participan en el traslado y la entrega de esos bienes a los consumidores finales. Se muestra un marco teórico que pone de manifiesto el contexto sobre la evolución del comercio electrónico, la importancia de la cadena de suministro así como de las empresas de logística haciendo hincapié en su contribución para dar certidumbre al cliente y que pueda lograrse su satisfacción y lealtad. Con relación a los resultados, se realizaron se presentaron comparaciones de las estrategias implementadas por las empresas minoristas en México tomando como referencias a Zara, HEB México y The Home Depot. También se contempló a las empresas de logística más importantes que operan en el país: UPS, DHL, Fedex y Estafeta y finalmente, se cotejaron las semejanzas y diferencias entre ambos tipos de empresas. Se encontraron diferencias importantes sobre todo entre las empresas minoristas en cuanto a los costos de envío y las políticas aplicables.

Palabras clave: Cadena de Suministro, Comercio Electrónico, Empresas de Logística, Empresas Minoristas.

\begin{abstract}
This study is an exploratory research on the strategies applied by retail companies that carry out electronic commerce and in this way mainly provide physical goods and the logistics companies that participate in the transfer and delivery of these goods to final consumers. A theoretical framework is shown that highlights the context on the evolution of electronic commerce, the importance of the supply chain as well as the logistics companies, emphasizing their contribution to give certainty to the client and that their satisfaction and loyalty can be achieved. Regarding the results, comparisons were made of the strategies implemented by the retail companies in Mexico, taking as references Zara, HEB México and The Home Depot. The most important logistics companies operating in the country were also considered: UPS, DHL, Fedex and Estafeta and finally, the similarities and differences between both types of companies were compared. Significant differences were found especially between retail companies in terms of shipping costs and applicable policies.
\end{abstract}

Keywords: E-Commerce, Logistic Companies, Retail Companies, Supply Chain.

JEL: M, M3, M31. 


\section{INTRODUCCION}

La evolución hacia nuevos formatos en los negocios se ha acelerado notablemente en los últimos años y como es de esperarse ante estos cambios, han emergido pioneros que se han desempeñado con mayor éxito, sentando un precedente que luego otros competidores vinieron a seguir y lo adaptaron a su contexto, siendo este un plan donde la actualización constante y enfoque a la satisfacción del cliente es imprescindible en este modelo de negocios.

Uno de los formatos que ha ido cobrando más fuerza ante la llegada de nuevas y mejores tecnologías, así como nuevas estrategias y herramientas de logística, es el comercio electrónico cuyos inicios datan según Ferreira dos Santos, Ricardo Sabino, Macedo Morais, \& Gon çalves, (2017) a partir de 1990, al notar las empresas los avances en las tecnologías de transmisión, procesamiento y almacenamiento, formularios de presentación, ergonomía, disponibilidad de información, rapidez y fiabilidad, y se observó una amplia gama de posibilidades, con especial énfasis en la comunicación, esto relacionado a la modalidad B2C ("business to consumer").

Ya que, según estos autores citando a Galinari et al. (2015) el comercio electrónico tuvo su primera fase sobre la década de 1970, cuando este se limitó a las operaciones entre grandes corporaciones qe establecieron entre ellas redes de comunicación privadas y, mediante sistemas electrónicos de transferencia de fondos, que realizaron electrónicamente transacciones financieras e intercambios de documentos.

Una de las claves del éxito de cualquier empresa es la búsqueda constante de la satisfacción del cliente y en el modelo de comercio electrónico no es la excepción, y sirva como ejemplo el trabajo de Zhang, Onal, Das, Helminsky, \& Das (2019) que identifican que el comercio en línea está dominado por Amazon, representando el
$43 \%$ de todas las ventas en línea estadounidenses en 2016. Numerosos informes de medios sugieren que el éxito de Amazon está impulsado por la nueva cadena de suministro de cumplimiento y logística que opera (Onal et al., 2017).

Entre los factores que podrían influir más en la satisfacción del cliente es la incertidumbre que surge a raíz del tiempo transcurrido entre la realización de un pedido y la recepción del producto solicitado.

Otro ejemplo de la industria es lo que proponen Pan, Giannikas, Han, Grover-Silva, \& Qiao, (2017) con relación al comercio electrónico de comestibles y la entrega a domicilio - el proceso de entrega de productos desde el punto de almacenamiento de un minorista (por ejemplo, centros de distribución, tiendas a la casa de un cliente) - desempeña un papel crucial (Punakivi y Saranen, 2001). (...) y se ha convertido en un canal de distribución dominante del comercio electrónico de negocio a consumidor (Campbell y Savelsbergh, 2006).

Adicional a esto, y derivado de un estudio anterior realizado por Aranda, Rebolledo \& Cantú (2019) en el cual se hizo la recomendación de realizar una investigación que profundice en el entorno que rodea tanto a la empresa vendedora como a la empresa distribuidora, en esta oportunidad se presenta un estudio exploratorio y descriptivo que muestra el ambiente querodea a estas empresas y las estrategias que han implementado para reducir la incertidumbre de sus clientes y así mantenersu satisfacción.

\subsection{HIPÓTESIS}

La hipótesis que guía a este estudio es: las empresas que realizan actividades de comercio electrónico dirigidas al consumidor (B2C) y las empresas de logística han implementado estrategias que contribuyen a incrementar la satisfacción del cliente. 


\subsection{OBJETIVO GENERAL}

El objetivo es identificar y conocer que estrategias han implementado empresas que realizan actividades de comercio electrónico y las empresas de logística que participan en la cadena de valor para llevar bienes y servicios al consumidor.

\subsection{OBJETIVOS ESPECÍFICOS}

- Identificar las estrategias que han implementado las empresas que realizan actividades de comercio electrónico y las empresas de logística.

- Conocer el impacto que han tenido estas estrategias en sus resultados.

\subsection{JUSTIFICACIÓN}

Este estudio busca dar continuidad al ya iniciado en el año 2019 en el que se exploró la perspectiva del cliente, exponiendo ahora que es lo que han hecho las empresas involucradas para contribuir a la reducción de la incertidumbre de sus clientes $y$ por consecuencia, a una mayor satisfacción lo que llevaría a una mayor lealtad y repetición de compras en el futuro, puesto que el alto costo por atraer nuevos clientes en Internet y la dificultad relativa para retenerlos hacen que la lealtad del cliente sea un activo esencial para muchas empresas de comercio electrónico. (Gefen, 2002)

\section{MARCO TEÓRICO}

\subsection{Comercio Electrónico}

El concepto de comercio electrónico lo define Pedro Barrientos, (2017) citando a Chaffey y Ellis-Chadwick (2014) como las transacciones financieras y de información realizadas de manera electrónica entre una organización y cualquier tercero con el que tenga tratos.

También, Ríos Ruiz (2015) define al comercio electrónico como el que se lleva a cabo a través de internet como cualquier forma de transacción o intercambio de información comercial basada en la transmisión de datos a través de esta red de comunicación.

Es así como, el comercio electrónico se ha convertido en una herramienta vital para empresas y consumidores de todo el mundo tanto para consumir productos y servicios en la vida diaria de los consumidores del siglo XXI (Sharma \& Lijuan, 2014)

Como se ha mencionado en la sección introductoria de este trabajo de investigación, el comercio electrónico ha sido tema de debate respecto a sus inicios como tal.

De acuerdo a la bibliografía consultada, se puede establecer que esta actividad tuvo sus inicios en los años 70, cuando se limitó a las operaciones entre grandes corporaciones que establecieron entre ellas redes de comunicación privadas y, mediante sistemas electrónicos de transferencia de fondos, realizaron electrónicamente transacciones financieras e intercambios de documentos. (Ferreira dos Santos, Ricardo Sabino, Macedo Morais, \& Gon çalves, 2017)

Aunque, Ariguzo, Mallach, \& White (2006) en su trabajo "The first decade of $e$ commerce" mencionan que existió una cierta euforia en el mercado ante los nuevos medios de hacer negocios y el nacimiento de una internet que era de enfoque general y más específicamente una World Wide Web accesible para aquellos con menor experiencia técnica y tecnológica (Connolly, 2000).

Del mismo modo, los autores Ariguzo, Mallach, \& White (2006) citan que la difusión desenfrenada y el éxito de Internet (...) y la aparente recompensa de la gran cantidad de inversión corporativa en tecnologías de la información en los EE. UU. y otras partes del mundo desde mediados de la década de 1980 hasta mediados de la década de 1990, llevó a muchos optimistas a declarar muerto el ciclo económico tradicional. Pero, este estado de euforia no fue sostenible por mucho tiempo. 
Por otro lado, otros autores proponen que los inicios como tal, fueron durante los años 90, trayendo consigo retos para las empresas ya que durante esos años, el inicio de la era digital como la conocemos, trajo consigo una incertidumbre a tal proporción que causó el efecto "dotcom bubble" que Morris \& Alam, (2012) mencionan citando a diversos autores que durante la burbuja de las puntocom en la década los 90, la valoración del mercado de renta variable fue un tema popular para los inversionistas, analistas financieros $\mathrm{y}$ académicos (...).

Mientras que, Penman (2003) describe el período de la burbuja económica de la década de 1990, como una reacción en cadena donde la inversión impulsiva desplazó a la inversión fundamental.

Pero, el estado de ánimo fue tal vez mejor descrito por Alan Greenspan, Ex-presidente de la Junta de la Reserva Federal, cuando advirtió sobre la situación en un discurso en the American Enterprise Institute for Public Policy Research el 5 de diciembre de 1996 (Greenspan, 1996).

En resumen, las empresas de comercio electrónico al nacer tuvieron un inicio difícil al presentarse retos nunca vistos ( Ljungqvist \& Wilhelm, Jr. , 2002).

\subsection{CADENA DE SUMINISTRO}

El objetivo general de la gestión de inventarios o cadena de suministro es garantizar la disponibilidad oportuna de los elementos que se necesitan (materia prima, materiales en proceso, productos terminados, insumos, repuestos, etc.), en las condiciones deseadas y en el lugar correcto. (Salas-Navarro, MaiguelMejía, \& Acevedo-Chedid, 2017)

Además, Salas-Navarro, Maiguel-Mejía, \& Acevedo-Chedid, (2017) definen a la cadena de suministro como aquellos miembros del canal de suministro más allá de los proveedores o de los clientes inmediatos de una empresa.
También mencionan que a lo largo de la historia se han identificado dos enfoques de la cadena de suministro:

i) Tradicional, vista como la interacción entre los sistemas totalmente integrados verticalmente y aquellos en los que cada miembro del canal opera de forma completamente independiente, así como la integración de procesos claves de negocio desde el usuario final por medio de proveedores que suministran productos, servicios e información; y

ii) Colaborativo, que se presenta cuando varios integrantes trabajan conjuntamente para planear y ejecutar actividades de la cadena de suministro con el fin de lograr una visión compartida de las oportunidades del mercado, consiguiendo más beneficios de los que pudieran conseguir si actuaran independientemente.

Por otro lado, Sengupta (2018) habla acerca de la cadena de suministro y propone que este sigue una cronología simple:

i) El cliente ordena un producto y paga el monto a la empresa vendedora.

ii) La empresa vendedora, mediante pago, despacha el producto a través de un proveedor de logística y

iii) El proveedor de logística acuerda un contrato de pago con el proveedor que consiste en la transferencia de bienes desde las instalaciones del vendedor a los clientes.

\subsection{EMPRESAS DE LOGÍSTICA}

Las empresas de logística cumplen una función de suma importancia puesto que estas permiten a las empresas proyectar dichas actividades en un escenario externo, visualizar las actividades y enlaces necesarios para comercializar los productos y elevar su competitividad en los niveles nacional o internacional. (Cano Olivos, Orue Carrasco, Martínez Flores, Mayett Moreno, \& López Nava, 2015) 
Por otra parte, Ballou (2004) menciona que la logística es la parte del proceso de la cadena de suministro que planea, lleva a cabo y controla el flujo y almacenamiento eficiente y efectivo de bienes y servicios, así como de la información relacionada, desde el punto de origen hasta el punto de consumo, con el fin de satisfacer los requerimientos de los clientes.

Dicho de esta forma, se puede definir a una empresa de logística como la que lleva a cabo las actividades de despacho y al personal que separa, consolida, carga y envía los pedidos de los clientes de una compañía, catalogándolas como rutinarias y no generadoras de valor agregado para el producto. (Mora García, 2016)

Además, tiene como función general la distribución física (el almacenamiento y el transporte), otros conceptos como la localización de las plantas y bodegas, los niveles de inventarios, los sistemas de indicadores de gestión y el sistema de información; los cuales se constituyen en aspectos importantes del proceso logístico integral. (Mora García, 2016)

De acuerdo a las definiciones del concepto de logística y la descripción de las actividades fundamentales de estas organizaciones, se puede inferir que la importancia de estas es substancial puesto que, sin ellas, el sistema de entregas a los consumidores finales se vería interrumpida.

Aun así, Meléndrez-Acosta (2018) menciona que, de acuerdo con Fernández, A. Hurtado, \& Peraluno (2005) uno de los problemas principales en la red tiene que ver con el proceso de logística, el transporte y el proceso de pago.

Torres Amaya (2007) menciona la importancia de la logística y las implicaciones negativas que tiene dentro del flujo de mercancías, ya que según un $19.5 \%$ de las compras realizadas por Internet en España no llegaron a entregarse y el $72 \%$ de los sitios no ofrece fecha de entrega aproximada.

\section{METODOLOGÍA}

Esta investigación es de tipo exploratoria ya que, de acuerdo con Behar Rivero (2008) este tipo de investigación tiene como propósito el destacar los aspectos fundamentales de una problemática determinada y encontrar los procedimientos adecuados para elaborar una investigación posterior. Además, gracias a este tipo de investigación se pueden abrir nuevas líneas de investigación y proceder a su consecuente comprobación.

Se identificaron organizaciones dentro del sector minorista y logística donde se han aplicado estrategias para reducir los niveles de incertidumbre, manteniendo o incluso aumentando la satisfacción del cliente, destacando los resultados que han producido.

Cabe precisar que no se busca ni pretende manipular las acciones de las empresas citadas en este estudio, debido a que no se forma parte de ellas y se refiere meramente a hechos ya realizados. Con esto se agrega valor a la postura de este estudio puesto que según Hernandez, Fernandez, \& Baptista (2006) sirven para familiarizarnos con fenómenos relativamente desconocidos, obtener información sobre la posibilidad de llevar a cabo una investigación más completa respecto de un contexto particular, investigar nuevos problemas, identificar conceptos, variables promisorias, establecer prioridades para investigaciones futuras, sugerir afirmaciones y postulados.

Además, esta investigación es de tipo descriptivo porque se detallan hechos ya efectuados por las organizaciones seleccionadas. De acuerdo con Behar Rivero (2008) el propósito de este tipo de investigación es:

1) Establecer las características demográficas de las unidades investigadas (número de población, distribución por edades, nivel de educación, etc.). 
2) Identificar formas de conducta, actitudes de las personas que se encuentran en el universo de investigación (comportamientos sociales, preferencias, etc.)

\section{3) Establecer comportamientos concretos.}

4) Descubrir y comprobar la posible asociación de las variables de investigación.

Por otro lado, Hernandez, Fernandez, \& Baptista, (2006) hablan al respecto y mencionan que un estudio descriptivo busca especificar propiedades, características y rasgos importantes de cualquier fenómeno que se analice y describa tendencias de un grupo o población.

También se considera un estudio de caso ya que de acuerdo con Stake (1999) se espera que abarque la complejidad de un caso particular. Chaverra Fernández, Gaviria Cortés, \& González Palacio, (2019) hacen referencia a Pérez (2008, p. 443) en su trabajo Investigación cualitativa. Retos e interrogantes donde mencionan que el estudio de casos se desarrolla en las situaciones reales en las que los individuos estudiados tienen responsabilidades y obligaciones con las que el estudio puede interferir.

Por lo que, al estudiar una empresa, se estaría cumpliendo con la descripción del concepto puesto que el caso puede ser una persona, un programa, un evento, un proceso, un grupo social. (Chaverra Fernández, Gaviria Cortés, \& González Palacio, 2019)

\section{RESULTADOS}

La comodidad es uno de los principales factores que promueven el uso del comercio electrónico entre consumidores y como se mencionó en apartados anteriores, el amplio desarrollo de las tecnologías de información, el internet y la accesibilidad de estas hoy en día hacen el comercio electrónico una opción fiable, sin embargo, las soluciones de logística no son igual de sencillas o accesibles.

\subsection{ESTRATEGIAS IMPLEMENTADAS POR EMPRESAS DE COMERCIO ELECTRONICO}

Garner (2018) menciona en su estudio que, Amazon comenzó en el año 1995 vendiendo exclusivamente libros y que, al primer año de operaciones, contaba con ventas en todo el territorio continental de Estados Unidos, así como también en 40 países del mundo. La empresa, además, menciona ha creado un estándar y expectativa en los consumidores alrededor del mundo; gracias a su servicio/estrategia de ofrecer sus envíos por medio de la membresía "Amazon Prime" los clientes pueden recibir cualquier producto (siempre y cuando la mercancía en cuestión aplique) en 2 días o menos.

Otra de las estrategias es cultivar una relación con vendedores, centros de distribución y otros servicios para que la empresa pueda tener capacidad de mejorar precios y servicio.

Una gestión efectiva de la logística puede ayudar a las empresas a obtener ventajas competitivas a través del valor agregado y reducción de costos. (Mckinnon, 2017)

Otro punto importante para resaltar es como las demás empresas llevan a cabo sus operaciones, como atienden las necesidades del cliente y como ofrecen certidumbre al momento en que se hace un pedido a través de sus plataformas.

La empresa ZARA del grupo Inditex (ZARA, s.f.), provee a sus clientes la capacidad de verificar los métodos de envío incluso antes de ver el catálogo que ofrece, aunque de forma poco informativa, puesto que el portal de internet menciona que las opciones de envío pueden variar en función de la dirección de entrega, la hora de compra y la disponibilidad de los artículos. Además, las opciones de envío aplicables se muestran al momento de la compra, por lo que, como se mencionó antes, no supone 
certidumbre ni atiende las posibles preferencias del consumidor respecto a las empresas de paquetería.

También, para el cliente implica un costo monetario por concepto de envío. Para el caso de México, la empresa menciona que este servicio tiene un costo de $\$ 55$ pesos mexicanos en pedidos menores a $\$ 899$ pesos, ya que, en los envíos con valor superior a éste, se anula el cargo; con un tiempo de entrega de 2 a 4 días hábiles sin opción a revisar quien envía la mercancía al momento de pagar el pedido.

Aunque si así prefiere el cliente, puede optar por un servicio de paquetería exprés, con un costo de $\$ 95$ pesos mexicanos independientemente del costo del pedido.

Otra opción que se ofrece es el recoger el pedido en tienda física. Pero la empresa no ofrece prontitud de entrega, anulando el beneficio ya que las mercancías llegarán a manos del cliente en un lapso mayor a 2 días hábiles.

Al mismo tiempo, HEB empresa minorista de origen estadounidense que comercia un amplio rango de productos a sus clientes, ofrece diversas opciones de entrega de los pedidos realizados.

En su página de internet, no existe un apartado como tal de información sobre envíos, aunque dentro de los términos y condiciones de servicio se menciona que existen 2 métodos de entrega de productos, el primero se promueve como "Pick \& Go" donde el cliente se presenta físicamente en tienda en un lugar designado para que sean entregados los productos. La otra opción es el tradicional envío a domicilio.

Respecto a esta última posibilidad, la información presentada es poco clara en términos generales ya que no se ubica fácilmente. El envío aplica solamente a algunas zonas de la ciudad, con un cargo de $\$ 49.00$ (cuarenta y nueve pesos 00/100 M.N.) incluyendo el Impuesto al Valor Agregado.
(H-E-B México, 2020) por concepto de entrega a domicilio.

Se visualiza que no se ofrece certidumbre en ninguno de los 2 métodos, puesto que los términos y condiciones mencionan que "HEB" realizará su mejor esfuerzo para llegar dentro del horario estipulado en la confirmación de compra; sin embargo, si existiera algún retraso, el cliente será notificado oportunamente por parte de sus socios (término con el que identifican a sus empleados), sin que esto implique incumplimiento de esos Términos y Condiciones ya que dependen de circunstancias como el tráfico, clima, marchas, protestas, entre otros. Por lo anterior, "HEB" en ningún caso se responsabiliza de dicho retraso. (H-E-B México, 2020)

Otro minorista, pero del sector de mejoras del hogar, Home Depot ofrece a sus clientes diversos métodos de entrega cuando se levantan pedidos a través de su portal. La página de internet ofrece información al respecto y siguiendo el mismo criterio, la empresa también ofrece envío gratuito en compras mayores de $\$ 499$ pesos mexicanos, lo cual puede ser un atractivo para las personas que adquieren productos como, ventiladores, electrodomésticos o materiales de construcción que se pueden adquirir en volumen por lo que muchos de sus clientes aprovechan el envío gratis (The Home Depot, 2019).

Sin embargo, las entregas están sujetas a un horario, que en México es de 8:00 a.m. a 7:00 p.m., dando discreción a las tiendas respecto a si realizan entregas dominicales, así como a zonas de difícil acceso (ej. Isla Mujeres o Cozumel), con un rango mínimo de 48 a 96 horas después de hacer el pedido en el portal.

En la información que la empresa menciona respecto a los cargos en categorías en las que no aplica promoción de envío gratis, los costos de envío varían por plaza y por categoría de producto. El costo de envío a Cozumel e Isla Mujeres incluye toda transportación marítima (Ferry) y varía 
dependiendo de la carga de los productos. A continuación, se presentan los rangos de costos sin IVA para zonas metropolitanas:

- Ciudad de México y Estado de México desde: $\$ 100.00$

- $\quad$ San José del Cabo desde: $\$ 138.00$

- Pachuca desde $\$ 100.00$

- Resto de la República desde $\$ 88.00$

(The Home Depot, 2019)

Por lo que al rastreo de envíos concierne, la empresa reporta que ofrece números de rastreo y actualizaciones en el portal y a través de notificaciones en el correo electrónico del cliente.

\subsection{ESTRATEGIAS IMPLEMENTADAS POR EMPRESAS DE LOGISTICA}

Del mismo modo, existen empresas de paquetería en México que ofrecen sus servicios, para comenzar, la empresa UPS (United Parcel Service) ofrece información detallada para quienes desean enviar paquetes a través de ellos.

De acuerdo al portal de (UPS, 2021), dentro de los tipos de servicios que ofrecen, existen 3 grandes rubros dirigidos al destino donde se envía el paquete y tiempo de entrega, la empresa los denomina como Servicio Nacional, Servicio Internacional 1 a 3 días, Servicio Internacional 2 a 7 días.

Como todas las empresas que se dedican al envío de paquetes, los precios se rigen por el peso de este. Los precios en sí varían considerablemente el rango de precios va desde $\$ 390.22$ pesos mexicanos para los paquetes en sobre o hasta 1 kilo, hasta 5,986.88 para los paquetes de 45 kilos en la zona más costosa del país.

La empresa divide al país en zonas, estas zonas varían de acuerdo con el estado y sus ciudades, por lo que es complejo mencionar a detalle sin embargo los datos se encuentran en su Guía de servicios y tarifas. Ofreciendo incluso la recolección del paquete al domicilio del remitente. La empresa ofrece el rastreo en su página de internet, además del servicio a cliente.

Por otro lado, la empresa alemana DHL ofrece herramientas personalizadas para cotizar el envío de paquetes a través de ellos. Sin embargo, no ofrece información al público en general, ya que requieren creación de una cuenta en su portal para poder acceder a sus herramientas. Si bien esto ofrece una ventaja a las empresas que recurrentemente envían productos a sus clientes o colaboradores, no ofrece certeza en cuanto al costo a remitentes ocasionales.

Sin embargo, la empresa es conocida por su amplia capacidad y entregas en tiempo y forma ya que ofrecen envíos de paquetes en sobres hasta pallets de mercancías. (DHL, 2021)

La empresa nacional Estafeta, ofrece distintos servicios para envío de mercancías, entre las opciones de mensajería nacional está: Día Siguiente, Dos Días, Terrestre, Múltiple, Cuenta Concentradora (dirigida a empresas) y servicios complementarios; así como exportaciones a Estados Unidos, Globales exprés e importaciones exprés. También ofrecen soluciones de logística del tipo aérea, LTL, Freight Forwarding y soluciones para empresas. (ESTAFETA, s.f)

Fedex, ofrece opciones similares a las empresas antes mencionadas, entre los servicios que ofrece están FedEx Express Nacional, FedEx Express Internacional, Soluciones multimodales a Estados Unidos. Las tarifas varían de la misma forma, ya que el territorio nacional está dividido en zonas por la empresa y cada zona supone un costo distinto a otras.

La empresa comparte los detalles de sus esquemas tarifarios en su portal de internet, sin embargo, si un cliente empresarial u ocasional busca hacer uso de sus servicios de mensajería, tiene como requisito crear una cuenta en el portal. (FEDEX, 2021) 
Tabla 1 Opciones de Envío y rastreo en empresas de logística

\begin{tabular}{|c|c|c|c|c|}
\hline $\begin{array}{l}\text { Opciones de } \\
\text { Envío y } \\
\text { Rastreo }\end{array}$ & UPS & Fedex & Estafeta & DHL \\
\hline $\begin{array}{l}\text { Opciones de } \\
\text { envío }\end{array}$ & $\begin{array}{l}\text { Directamente en sucursal, } \\
\text { recolección a domicilio de } \\
\text { paquete. * puede aplicar una } \\
\text { tarifa por combustible extra. }\end{array}$ & $\begin{array}{l}\text { Directamente en } \\
\text { sucursal, recolección } \\
\text { a domicilio de } \\
\text { paquete. }\end{array}$ & $\begin{array}{l}\text { Directamente en } \\
\text { sucursal, recolección } \\
\text { a domicilio de } \\
\text { paquete. *solo } \\
\text { clientes usuales o } \\
\text { alto volumen }\end{array}$ & $\begin{array}{l}\text { Directamente en } \\
\text { sucursal, recolección } \\
\text { a domicilio de } \\
\text { paquete. }\end{array}$ \\
\hline $\begin{array}{l}\text { Costo por } \\
\text { envío }\end{array}$ & $\begin{array}{l}\text { El costo varía por peso del } \\
\text { paquete a enviar. }\end{array}$ & $\begin{array}{l}\text { El costo varía por } \\
\text { peso del paquete a } \\
\text { enviar. }\end{array}$ & $\begin{array}{l}\text { El costo varía por } \\
\text { peso del paquete a } \\
\text { enviar. }\end{array}$ & $\begin{array}{l}\text { El costo varía por } \\
\text { peso del paquete a } \\
\text { enviar. }\end{array}$ \\
\hline $\begin{array}{l}\text { Opciones de } \\
\text { rastreo de } \\
\text { paquete }\end{array}$ & $\begin{array}{l}\text { La empresa ofrece rastreo } \\
\text { por medio de la página } \\
\text { principal, en la herramienta } \\
\text { de rastreo. Servicio a cliente } \\
\text { por teléfono o e-mail }\end{array}$ & $\begin{array}{l}\text { La empresa ofrece } \\
\text { rastreo por medio de } \\
\text { la página principal, } \\
\text { en la herramienta de } \\
\text { rastreo. Servicio al } \\
\text { cliente por teléfono o } \\
\text { e-mail }\end{array}$ & $\begin{array}{l}\text { La empresa ofrece } \\
\text { rastreo por medio de } \\
\text { la página principal, } \\
\text { en la herramienta de } \\
\text { rastreo. Servicio al } \\
\text { cliente por teléfono o } \\
\text { e-mail }\end{array}$ & $\begin{array}{l}\text { La empresa ofrece } \\
\text { rastreo por medio de } \\
\text { la página principal, } \\
\text { en la herramienta de } \\
\text { rastreo. Servicio al } \\
\text { cliente por teléfono o } \\
\text { e-mail }\end{array}$ \\
\hline $\begin{array}{l}\text { Servicio } \\
\text { internacional }\end{array}$ & $\begin{array}{l}\text { Sí ofrece servicio de } \\
\text { mensajería internacional }\end{array}$ & $\begin{array}{l}\text { Sí ofrece servicio de } \\
\text { mensajería } \\
\text { internacional }\end{array}$ & $\begin{array}{l}\text { Sí ofrece servicio de } \\
\text { mensajería } \\
\text { internacional }\end{array}$ & $\begin{array}{l}\text { Sí ofrece servicio de } \\
\text { mensajería } \\
\text { internacional }\end{array}$ \\
\hline
\end{tabular}

Fuente: (elaboración propia) 
Tabla 2 Opciones de rastreo y envío de empresas de comercio electrónico

\begin{tabular}{|c|c|c|c|}
\hline $\begin{array}{l}\text { Opciones De } \\
\text { Envío Y } \\
\text { Rastreo } \\
\end{array}$ & H-E-B México & ZARA México & The Home Depot México \\
\hline $\begin{array}{l}\text { Opciones de } \\
\text { envío y/o } \\
\text { recolección } \\
\text { en tienda. }\end{array}$ & $\begin{array}{l}\text { Sí. Ofrece envío a } \\
\text { domicilio de mercancías, } \\
\text { así como recolección en } \\
\text { tiendas. }\end{array}$ & $\begin{array}{l}\text { Sí. Ofrece envío a domicilio de } \\
\text { mercancías, así como recolección } \\
\text { en tiendas, aunque es variable la } \\
\text { recolección, puede tardar de } 2 \text { a } 3 \\
\text { días hábiles la recolección en } \\
\text { tienda. }\end{array}$ & $\begin{array}{l}\text { Sí. Ofrece envío a domicilio de } \\
\text { mercancías, así como recolección } \\
\text { en tiendas. }\end{array}$ \\
\hline $\begin{array}{l}\text { Costo por } \\
\text { envío (si } \\
\text { aplica) }\end{array}$ & \$49 MXN & $\begin{array}{l}\$ 55 \text { MXN en pedidos menores a } \\
\$ 899 . \text { Mayores a } \$ 899 \text { el envío es } \\
\text { gratis. } \\
\text { Opción de envío exprés por } \$ 99\end{array}$ & $\begin{array}{c}\text { Envío gratis en pedidos mayores } \\
\text { de } \$ 499 . \text { En los casos que aplica } \\
\text { los costos son: Ciudad de } \\
\text { México y Estado de México } \\
\text { desde: } \$ 100.00 ; \text { San José del } \\
\text { Cabo desde: } \$ 138.00 ; \text { Pachuca } \\
\text { desde: } \$ 100.00 ; \text { Resto de la } \\
\text { República desde: } \$ 88.00\end{array}$ \\
\hline $\begin{array}{l}\text { Opciones de } \\
\text { rastreo de } \\
\text { paquete }\end{array}$ & $\begin{array}{l}\text { No como tal. Ofrece sus } \\
\text { envíos en un rango de } \\
\text { horario, sujeto a retrasos }\end{array}$ & $\begin{array}{l}\text { La empresa envía un número de } \\
\text { rastreo por correo electrónico. }\end{array}$ & $\begin{array}{c}\text { No ofrece opciones de rastreo de } \\
\text { envío. }\end{array}$ \\
\hline Devoluciones & $\begin{array}{l}30 \text { días posteriores a la } \\
\text { compra. Y } 7 \text { días en } \\
\text { artículos seleccionados. } \\
\text { no aplican en productos } \\
\text { perecederos, medicinas, } \\
\text { vinos, licores, ropa } \\
\text { interior, así como en } \\
\text { muebles }\end{array}$ & $\begin{array}{c}30 \text { días desde la fecha de envío de } \\
\text { tu pedido para hacerlo de forma } \\
\text { gratuita. Los artículos deben } \\
\text { conservar todas las etiquetas y } \\
\text { estar en perfecto estado. Esto } \\
\text { puede ser en tienda o punto de } \\
\text { entrega }\end{array}$ & $\begin{array}{l}\text { Se tienen } 50 \text { días a partir de la } \\
\text { fecha de compra para realizar el } \\
\text { reembolso o cambio de mercancía } \\
\text { en la tienda donde se realizó la } \\
\text { compra. }\end{array}$ \\
\hline
\end{tabular}


Tabla 3

Comparativa de opciones de envío de empresas de logística y comercio electrónico

\begin{tabular}{|c|c|c|}
\hline Opciones de envío y rastreo & Empresas de Logística & Empresas de Comercio Electrónico \\
\hline Solución de envío y/o recolección. & $\begin{array}{l}\text { Por lo general las empresas ofrecen } \\
\text { recolectar a domicilio los paquetes } \\
\text { así mismo acepta dejar envíos en } \\
\text { sus sucursales. }\end{array}$ & $\begin{array}{l}\text { Se ofrecen envíos a domicilio y } \\
\text { recolección de parte del cliente en } \\
\text { tiendas físicas. }\end{array}$ \\
\hline Costo por envío (si aplica) & $\begin{array}{l}\text { Dada la naturaleza de las empresas, } \\
\text { estas cobran tarifas de acuerdo con } \\
\text { el peso del paquete y zona } \\
\text { geográfica. Además del tipo de } \\
\text { envío (exprés o económico) }\end{array}$ & $\begin{array}{l}\text { La mayoría de las empresas } \\
\text { analizadas, cargan a sus clientes con } \\
\text { un costo de envío, sin embargo, } \\
\text { anulaban el cargo si se cumplía con } \\
\text { un mínimo de compra. En el caso de } \\
\text { HEB no existe tal condición. }\end{array}$ \\
\hline Opciones de rastreo de paquete & $\begin{array}{l}\text { Sí. Estas empresas ofrecen } \\
\text { herramientas en internet y } \\
\text { aplicaciones móviles para rastrear } \\
\text { los envíos. }\end{array}$ & $\begin{array}{l}\text { No especifican exactamente como } \\
\text { informan del estatus de envío. }\end{array}$ \\
\hline Servicio internacional & $\begin{array}{l}\text { Estas empresas por su naturaleza y } \\
\text { alcance internacional proveen } \\
\text { servicios de mensajería } \\
\text { internacional y algunos hasta de } \\
\text { forma multimodal. }\end{array}$ & $\begin{array}{l}\text { Las empresas analizadas en este } \\
\text { estudio tienen sus filiales en México } \\
\text { por lo que no implica un uso de } \\
\text { envíos internacionales. }\end{array}$ \\
\hline Ayuda y Soporte & $\begin{array}{l}\text { Las empresas ofrecen atención vía } \\
\text { internet, correo electrónico y por } \\
\text { teléfono. Además de redes sociales } \\
\text { y documentos y sitios web con } \\
\text { preguntas frecuentes. }\end{array}$ & $\begin{array}{l}\text { Las empresas ofrecen atención vía } \\
\text { internet, correo electrónico, por } \\
\text { teléfono, redes sociales y chat. }\end{array}$ \\
\hline
\end{tabular}

Fuente: (Elaboración propia) 


\section{CONCLUSIONES}

Conforme a los resultados obtenidos de las empresas analizadas en esta investigación se puede concluir que en especial las empresas minoristas que realizan actividades de comercio electrónico han implementado estrategias para brindar opciones de compra y entrega a sus clientes y así mantener o incrementar su satisfacción y reducir su incertidumbre. En el caso de las empresas de logística también se identifican las estrategias de servicio que buscan contribuir al buen funcionamiento de la cadena de suministro y a la conformidad del cliente.

De acuerdo a la literatura revisada, se pudo identificar que el comercio electrónico ha ido evolucionando a través del tiempo hasta convertirse en lo que es actualmente y las empresas minoristas a nivel mundial lo han ido adoptando de forma paulatina (especialmente en México) aunque circunstancias extraordinarias vividas de forma global las han obligado a acelerar e innovar sobre la marcha dando como resultado lo que se detalla en la sección de resultados respecto a lo que hacen Zara, HEB y Home Depot en cuanto a las opciones de entrega que ofrecen para compras realizadas en línea así como las posibilidades de rastreo e información respecto a la ubicación además de la fecha y hora compromiso para recibir o recoger su compra según la opción elegida.

En cuanto a las empresas de logística todas ofrecen atención tanto en sucursal como recolección a domicilio de envíos y las diferencias que existen son en cuanto a posibles cargos adicionales por combustible extra (UPS) o preferencia en cuanto a recolección a domicilio para clientes frecuentes o que manejen altos volúmenes (Estafeta) quedando descartada esta posibilidad para el resto de los clientes.

También se aprecia que, al menos en cuanto a lo que refleja la literatura revisada, la cadena de suministro sigue un enfoque más bien tradicional y no colaborativo por lo que se sugiere la posibilidad de hacer un estudio más profundo el cual podría ser de campo para revisar si existe colaboración estrecha entre empresas minoristas y de logística que no se ha manifestado públicamente o se corrobora que siguen un enfoque completamente tradicional.

Lo anterior podría contribuir a explicar porque empresas como HEB México o Home Depot no ofrecen la posibilidad de rastrear la ubicación de los pedidos a sus clientes ni claridad de quien o que empresa se encargará de realizar la entrega. También esto pudiera verse reflejado en el caso de Zara en cuanto al tiempo en el que un cliente puede recoger en tienda su pedido (2 a 3 días hábiles) cuando pudiera ser en unas horas si la mercancía está disponible de forma inmediata en dicha sucursal.

También cabe precisar que hasta el momento no se ha encontrado información sobre estrategias que han implementado las empresas de logística en aspectos como atención al cliente, mayor efectividad en sus tiempos de entrega, vinculación con empresas minoristas para ofrecer un servicio integral, etc.

El presente estudio puede aportar información para conocer las estrategias que han implementado dos de los integrantes más importantes de la cadena de suministro y que inciden en la búsqueda de una mayor satisfacción del cliente así como de su lealtad. Y también se aprecia la posibilidad de futuros estudios que profundicen en dichas estrategias así como en la factibilidad de un enfoque colaborativo entre empresas minoristas y de logística para incrementar la efectividad y la certeza de que los pedidos serán entregados al cliente en tiempo y forma. 


\section{REFERENCIAS}

Ljungqvist, A. P., \& Wilhelm, Jr., W. J. (2002). IPO pricing in the dot-com bubble. Seminar presentations at the University of Florida and Oxford University, 1-40.

Ariguzo, G. C., Mallach, E. G., \& White, D. S. (2006). The first decade of e-commerce. Int. J. Business Information Systems, 239-255.

Ballou, R. (2004). Logística: administración de la cadena de suministro (5a). México: Prentice Hall.

Behar Rivero, D. S. (2008). Metodología de la Investigación. s.d.: Editorial Shalom.

Cano Olivos, P., Orue Carrasco, F., Martínez Flores, J., Mayett Moreno, Y., \& López Nava, G. (2015). Modelo de gestión logística para pequeñas y medianas empresas en México. Contaduría y Administración 60, 181-203.

Chaverra Fernández, B., Gaviria Cortés, D. F., \& González Palacio, E. V. (2019). El estudio de caso como alternativa metodológica en la investigación en educación física, deporte y actividad física. Conceptualización y aplicación. Retos, 35, 422427.

DHL. (2021). OBTENGA UNA COTIZACIÓN. Obtenido de DHL: https://www.dhl.com/mx-es/home/obtenga-una-cotizacion/one-time-cotizacion-deenvios.html

ESTAFETA. (s.f). ESTAFETA. Obtenido de ESTAFETA: https://www.estafeta.com/

FEDEX. (2021). Servicios y tarifas. Obtenido de México 2021:

https://www.fedex.com/content/dam/fedex/international/rates/fedex-rates-all-es-mx2021.pdf

Ferreira dos Santos, V., Ricardo Sabino, L., Macedo Morais, G., \& Gon çalves, C. (2017). E-Commerce: A Short History Follow-up on Possible Trends. International Journal of Business Administration, 130-138.

Garner, B. A. (2018). Amazon in the Global Market. Journal of Marketing and Management, 9 (2), 63-73.

Gefen, D. (2002). Customer Loyalty in E-Commerce. Journal of the Association for Information Systems, Volume 3, 27-51.

H-E-B México. (2020). Heb.com.mx. Obtenido de Términos y Condiciones: https://www.heb.com.mx/terminos-condiciones

Hernandez Sampieri, R., Fernandez-Collado, C., \& Baptista Lucio, P. (2006). Metodología de la Investigación. Mexico D.F.: McGraw Hill Interamericana.

Mckinnon, A. (2017). Handbook of Logistics and Supply-Chain Management. Integrated Logistics Strategies, 157-170. 
Meléndrez-Acosta, V. (2018). Logística del comercio electrónico: cross docking, merge in transit, drop shipping y click and collect. Científica, vol. 22, núm. 2, 105-112.

Mora García, L. A. (2016). Gestión Logística Integral: las mejores practicas en la cadena de abastecimiento . Ecoe Ediciones.

Morris, J. J., \& Alam, P. (2012). Value relevance and the dot-com bubble of the 1990s. The Quarterly Review of Economics and Finance, 243-255.

Pan, S., Giannikas, V., Han, Y., Grover-Silva, E., \& Qiao, B. (2017). Using customerrelated data to enhance e-grocery home delivery. Industrial Management \& Data Systems, 1917-1933.

Pedro Barrientos, F. (2017). Marketing + internet = e-commerce: oportunidades y desafíos. Finanz. polit. econ, 41-56.

Ríos Ruiz, A. d. (2015). ANÁLISIS Y PERSPECTIVAS DEL COMERCIO ELECTRÓNICO EN MÉXICO. Perfiles de las Ciencias Sociales, Año 3, Núm. 5, 61-87.

Salas-Navarro, K., Maiguel-Mejía, H., \& Acevedo-Chedid, J. (2017). Metodología de Gestión de Inventarios para determinar los niveles de integración y colaboración en una cadena de suministro. Ingeniare. Revista chilena de ingeniería, vol. $25 N^{o} 2$, 326-337.

Sengupta, S. N. (2018). Quintessence of third party (3PL) logistics. Journal of Global Operations and Strategic Sourcing, 11(2), 146-173.

Sharma, G., \& Lijuan, W. (2014). Ethical perspectives on e-commerce: an empirical investigation. Internet Research, 414-435.

Stake, R. E. (1999). Investigación con estudio de casos. Madrid: EDICIONES MORATA, S. L. .

The Home Depot. (01 de Enero de 2019). The Home Depot. Obtenido de CONDICIONES DE ENVÍOS EN TIENDA: https://www.homedepot.com.mx/condiciones-envios

Torres Amaya, E. (2007). Implicaciones de la logística en el comercio electrónico sobre la satisfacción del cliente final interactivo. Télématique, vol. 6, núm. 1, 208-223.

UPS. (28 de diciembre de 2021). Guia de tarifas y servicios. Obtenido de https://upscontentcentre.com/pdf/mx/es

ZARA. (2021). Zara.com. Obtenido de ENVÍO: https://www.zara.com/mx/es/help/metodos-de-envio-y-coste-h32.html

Zhang, J., Onal, S., Das, R., Helminsky, A., \& Das, S. (2019). Fulfilment time performance of online retailers - an empirical analysis. International Journal of Retail \& Distribution Management, 493-510. 
